\title{
Hardware para interfaz cerebro computador
}

\author{
Brain computer interface hardware.
}

\author{
Msc. Paulo Andrés Vélez Ángel, Phd. Juan Diego Gomez, Ing. Hector Garcia Arana \\ Centro de Bioinformatica y Biologia computacional BIOS - Universidad del valle, Tulua,, Colombia \\ $\frac{\text { paulo.velezecorreounivalle.edu.co }- \text { juan.gomez@bios.co }}{\text { hector.garciadcorreounivalle.edu.co }}$
}

\begin{abstract}
Resumen - Las interfaces cerebro computador, han empezado a verse en múltiples aplicaciones. Existen dispositivos comerciales para ser utilizados como sistemas de captura para esta tecnología, el problema radica en los costos de los mismos. En este artículo, se presenta la construcción de un equipo de captura de electroencefalografía de bajo costo que se conecta al computador para poder ser utilizado con este fin.
\end{abstract}

Palabras clave- Biopotenciales, Interfaces cerebro computador, Arduino.

Abstract - Brain computer interfaces are being increasingly mainstreamed in virtually all tech apps.

In line with this boom, the emergence of comercial devices for capturing brain activity continue to grow, although tightly associated to expensiveness.

Accordingly, this paper depicts the setting up of a low-cost, yet very efficient plug-and-play prototype to this end.

Key Word - Biopotentials, brain computer interfaces, Arduino.

\section{INTRODUCCIÓN}

Las interfaces cerebro - computador basadas en EEG, aparecieron hace varios años, aunque no sean muy populares aún. La principal desventaja que han tenido para ser populares, es el nivel de precisión que requiere la captura de señales electroencefalográficas, que son la materia prima que se utiliza para obtener las órdenes que se quieren convertir en acciones a realizar.

Para el diseño de este equipo, se utilizó como referencia el módulo EKG SHIELD, que es un módulo compatible con la plataforma Arduino y permite capturar señales electrocardiográficas y de electromiografía. Estas señales son más fáciles de capturar debido a que su potencial eléctrico es del orden de los milivolts, comparado con las señales del cerebro que son de microvolts.

Los investigadores del Semillero de Investigación de Tecnología Electrónica de la Universidad del Valle Sede
Tuluá (SITE) y del Centro de Bioinformatica y Biologia computacional BIOS, tomaron esta tarjeta y le incorporaron una ganancia de 1000 veces, para permitir amplificar con este mismo equipo, las señales provenientes del cuero cabelludo de un ser humano. Una vez obtenida esta señal, fue aplicada a la tarjeta Arduino UNO, que permitió la capacidad de procesamiento básico de la señal usando el algoritmo de la transformada rápida de Fourier, para obtener los componentes de frecuencia de la señal capturada y finalmente ser enviada a un sistema de cómputo, utilizando el puerto USB. En el computador, se implementó una aplicación que lee la señal y la puede capturar, para posteriormente ser utilizada como control de algún sistema.

\section{IMPLEMENTACION}

Las interfaces cerebro computador (BCI), son un nuevo tipo de periféricos, que permiten controlar los equipos de cómputo u otro tipo de equipos electrónicos, utilizando como señal de entrada la actividad eléctrica del cerebro.

Este proyecto pretende diseñar y construir un equipo versátil que pueda ser empleado para desarrollar aplicaciones en este nuevo campo de la tecnología, con materiales nacionales y a precios accesibles, principalmente orientadas al sector Salud (apoyando tratamientos para personas con discapacidad física) y también en beneficio del componente pedagógico de los estudiantes de los Programas Académicos de Tecnología en Electrónica, Tecnología en Sistemas e Ingeniería de Sistemas de la Universidad del Valle sede Tuluá, a través de lo cual se contribuye significativamente al desarrollo socioeconómico regional. De forma complementaria, es de considerar que si se diseña y construye la interfaz propia, con componentes de relativa facilidad de adquisición en el mercado local, esta tecnología puede ser empleada de una forma didáctica, complementando la estructura curricular de los programas académicos mencionados.

Con base en lo anterior, el desarrollo de esta tecnología y el uso de este equipo, se constituye en una herramienta importante para la estructuración y realización de un curso electivo dirigido a los estudiantes de los programas 
académicos mencionados anteriormente, que pueda contener además de un componente teórico, un componente práctico, con lo cual los futuros profesionales de la sede Tuluá en estos campos del conocimiento, cuentan con mayores fortalezas para facilitar su inserción en el campo laboral o la creación de nuevas empresas en el área tecnológica.

\section{A. Biopotenciales}

En el interior del cuerpo humano, se producen diferencias de potencial medidas entre el interior y exterior de la célula (a través de la membrana celular), estos son los biopotenciales. Existen muchos tipos de biopotenciales que dependen de las células que los genera y el organismo en que se encuentre entre los más conocidos se tiene:

- ECG (ELECTROCARDIOGRAMA): es de los más usados y mide la actividad eléctrica del corazón. Del orden de los milivolts.

- EEG (ELECTROENCEFALOGRAMA): Esta es la medida de la actividad eléctrica del cerebro. Del orden de los microvolts.

- $\quad$ EMG (ELECTROMIOGRAFIA): Esta mide la actividad eléctrica de los músculos y puede ser utilizada para el control de prótesis en discapacitados. Del orden de los milivolts.

- $\quad$ EGG (ELECTROGASTROGRAMA): Es la medición de los cambios eléctricos que se producen en la musculatura del estómago. Del orden de los milivolts.

- EOG (ELECTROOCULOGRAFIA): Potenciales eléctricos generados debido al movimiento de los globos oculares. Del orden de los milivolts.

\section{B. Estados mentales}

Las señales electroencefalografías, se pueden clasificar según la frecuencia de la misma en diferentes tipos, que se van a producir según el estado mental en que se encuentre el usuario de esta forma.

\section{Delta}

Señal de 10 a 50 micro voltios y una frecuencia de 0,2 a 3,5 Hz. (Ver Figura 1). Corresponde a un estado hipnótico, trabajo del hemisferio cerebral derecho en plena actividad, sueño profundo y meditación.

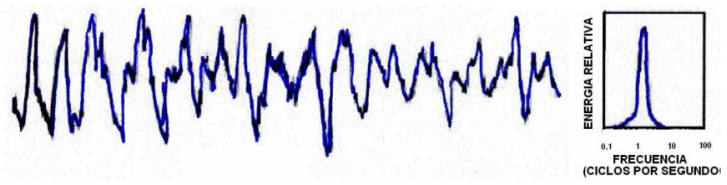

Figura 1. Onda Delta

\section{Theta}

Señal de 50 a 100 micro voltios de 3,5 a 7,5 Hz. Se puede ver en la figura 2 y corresponde a un estado de vigilia, equilibrio entre los hemisferios izquierdo y derecho.

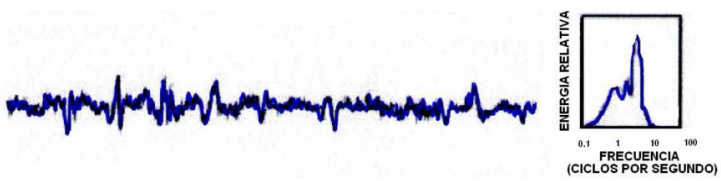

Figura 2. Onda Theta

\section{Alfa}

Señal de 100 a 150 microvoltios entre 7,5 a $13 \mathrm{~Hz}$. Se puede ver en la figura 3 y corresponde a un estado de relajación, tranquilidad, creatividad inicio de actividad plena del hemisferio izquierdo y desconexión del hemisferio derecho.

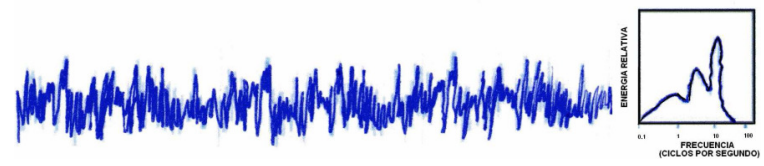

Figura 3. Onda Alfa

\section{4. $\mathrm{Mu}$}

Las ondas Mu (ver Figura 4), son ondas espontáneas que se encuentran en la banda de frecuencia entre 8 $\mathrm{Hz}$ y $13 \mathrm{~Hz}$, igual que las ondas Alfa. Ellas se registran principalmente en la zona Sensorio-motora (ver figura 1) y se registran en la posición $\mathrm{C}$ del sistema de colocación de electrodos (ver figura 2), manifestando una atenuación durante el movimiento o intento de movimiento de extremidades.
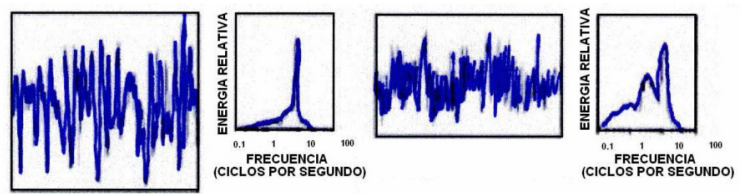

Figura 4. Onda $\mathrm{Mu}$

\section{Beta}

Señal de 150 a 200 micro voltios con frecuencias de 13 a $28 \mathrm{~Hz}$ (ver figura 5) Corresponde a estado de alerta máxima, vigilante, miedo, es la situación normal

cuando estamos despiertos, conduciendo, o trabajando en donde estamos en estado de alerta, ansiedad. 


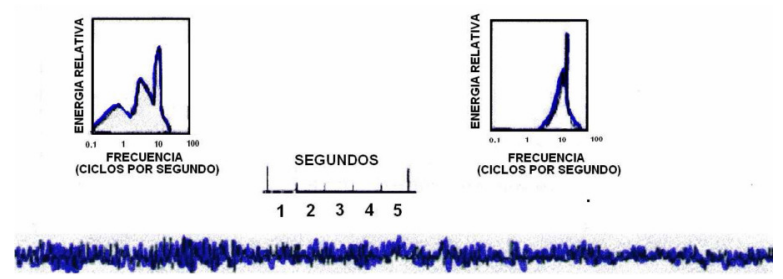

Figura 5. Onda Beta

\section{Ram-alta}

Señal superior a los 200 micro voltios y frecuencias mayores de $28 \mathrm{~Hz}$.

Corresponde a estados de stress y confusión.

\section{Ondas Gama}

Las ondas Gama se encuentran desde los $30 \mathrm{~Hz}$ hacia arriba. Se piensa que éstas reflejan el mecanismo subyacente de la conciencia. Se ha observado que las ondas Gama están asociadas a procesos de comunicación, feedback y sincronización entre las distintas zonas neuronales - módulos cerebrales durante la realización de actividades mentales complejas.

\section{Hardware}

\section{Electrodos}

Los electrodos[1] son los dispositivos que convierten las corrientes ionicas presentes en los organismos vivos en corrientes de electrones que son las que pueden circular por los conductores metálicos. Esto se puede observar en la Figura 6.

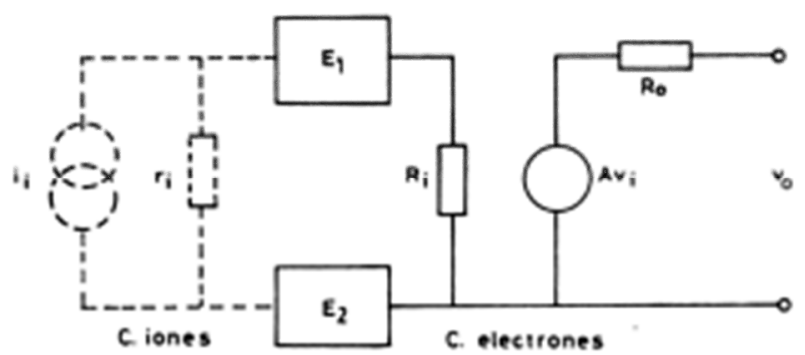

Figura 6. Modelo simplificado interfaces paciente - electrodoamplificador.

Los electrodos utilizados para el circuito, fueron superficiales, debido a que la captura de la señal sobre el cuero cabelludo es no invasiva.

\section{Amplificadores de instrumentación.}

Un amplificador de instrumentación [2] es un dispositivo creado a partir de amplificadores operacionales. Está diseñado para tener una alta impedancia de entrada y un alto rechazo al modo común (CMRR). Estas son condiciones óptimas para ser utilizado en circuitos que deben tener contacto con seres vivos. Además su utilización es común en aparatos que trabajan con señales muy débiles, tales como equipos médicos (por ejemplo, el electrocardiógrafo), para minimizar el error de medida.

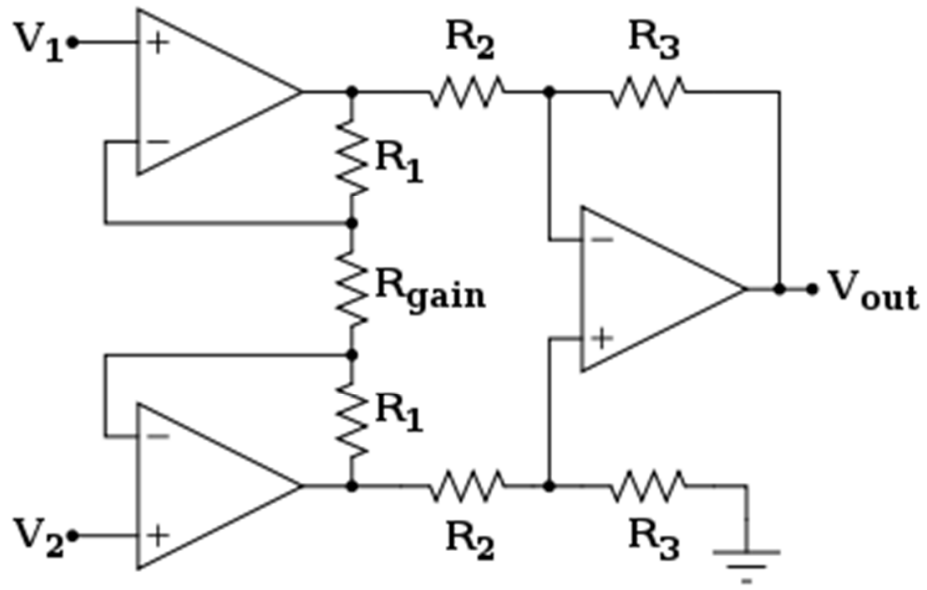

Figura 7. Amplificador de instrumentación

Se puede construir a base de componentes discretos como se muestra en la figura No 7 o se puede encontrar encapsulado (por ejemplo el AD620).

La operación que realiza es la resta de sus dos entradas multiplicada por un factor.

\section{Filtros pasa banda}

Las señales electroencefalografícas que son las que se van a utilizar para realizar control, presentan frecuencias desde los 1 $\mathrm{Hz}$ hasta los $100 \mathrm{~Hz}$, en varios estados mentales (Delta, Alfa, Betha y Gamma). Por esta razón se hizo necesaria la fabricación de un circuito que hiciera esta tarea además de tener un filtro rechaza banda de $60 \mathrm{~Hz}$. El filtro diseñado es un filtro Butterword de orden 4.

\section{Arduino}

Arduino es una plataforma de hardware libre, basada en una placa con un microcontrolador y un entorno de desarrollo, diseñada para facilitar el uso de la electrónica en proyectos multidisciplinares. Se ha hecho muy famoso en tiempos recientes, debido a la practicidad para el desarrollo de aplicaciones embebidas con él. Incorpora un lenguaje de programación de los periféricos conectados al microcontrolador, que hace que el desarrollo de los mismos, sea muy rápido.

\section{Conexión usb}

El Bus Universal en Serie (BUS) (en inglés: Universal Serial Bus), más conocido por la sigla USB, es un bus estándar industrial que define los cables, conectores y protocolos usados en un bus para conectar, comunicar y proveer de alimentación eléctrica entre computadoras, periféricos y dispositivos electrónicos. El sistema arduino, incorpora un puerto usb que puede ser utilizado para programar la plataforma, así como para ser usada como puerto de entrada salida. Dicho puerto, fue utilizado para el envió de los datos 
del sistema de captura hasta el PC y allí poder ser utilizados por el software.

\section{Software}

Para el uso de las señales capturadas, se desarrolló una aplicación en Visual Studio y en concreto en el lenguaje C\#. Esta aplicación va a ser utilizada para las futuras aplicaciones del equipo construido.

\section{Lectura puertos}

Inicialmente se estableció la conexión de captura de la información del puerto USB con el lenguaje y la interfaz de Arduino. A este se le generaron ciertos cambios, pasando los baudios de 9600 a 14400 ya que era más fluida la información.

A partir de esta lectura, se captura las señales mandadas por el arduino a través del puerto usb, lo cual se logra analizando si se está abierto o no el puerto serial para realizar la captura y a continuación se visualizara en el código.

En la codificación se realizaron capturas de excepción debido a que al abrir la conexión para el puerto usb, la aplicación se bloquea e impide la visualización y captura de la información, afectando totalmente la funcionalidad de la aplicación, pues se deseaba visualizar en tiempo real la señal capturada.

\section{Graficar señal}

Para poder visualizar la señal en una interfaz de usuario amigable, se recurrio a las librerías PlotLab y SignaLab de Mitov.

SignalLab es una librería que permite la manipulación de señales complejas rápidas y manejar su visualización.

PlotLab, ayuda a construir un trazado rápido de datos.

\section{Análisis espectral}

Utilizando el toolbox de SignalLab, fue posible implementar funciones que capturan la señal y mostraran los componentes de la transformada rápida de Fourier, para identificar en primera instancia los estados mentales del usuario, esta medida puede ser utilizada para un sistema de control que detecte dichos estados y genere acciones de control.

\section{Resultados obtenidos}

Utilizando un método analítico, se encontró que para medir la actividad eléctrica del cerebro, era necesario construir un equipo que capturara la información desde los electrodos de entrada y esta fuera pasada a un sistema de filtrado que permitiera eliminar los componentes que pueden considerarse como ruido o en la medida de señales electroencefalográficas, son conocidos como artefactos. En la figura No 8 se puede observar el diagrama esquemático del circuito correspondiente a la etapa de captura y filtrado de la señal EEG.

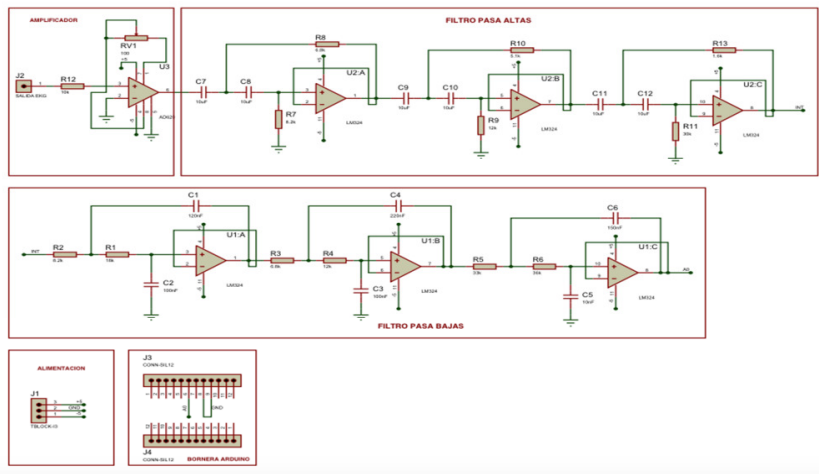

Figura 8. Diagrama esquemático hardware.

Se evaluó la captura de la información en tiempo real, conectando la plataforma arduino al circuito para verificar su conectividad y en la Figura No 9 se puede observar la interfaz construida en Visual Studio. El osciloscopio visualizado es un elemento de la librería PlotLab. Este se uso dos veces, una para mostrar la señal en el dominio del tiempo y la otra para representar la transformada de Fourier de la misma.

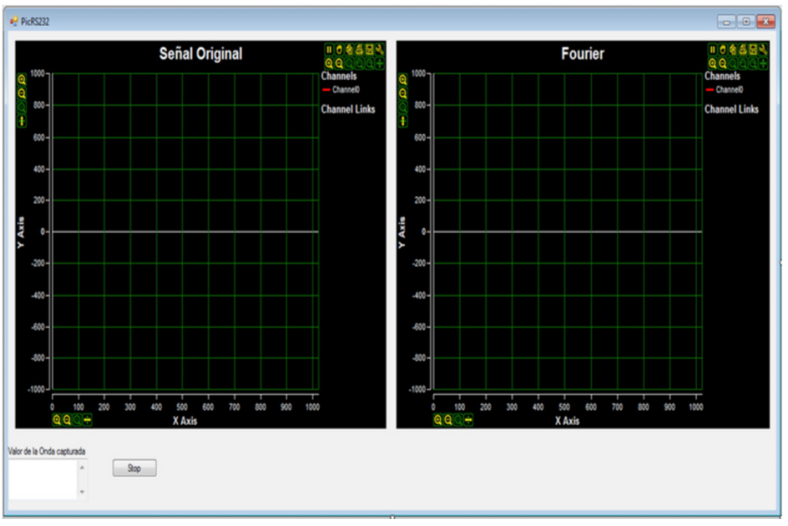

Figura 9. Interfaz de la aplicación.

Las figuras 10 y 11, muestran el circuito presentado funcionando en el laboratorio de electrónica de la universidad del Valle - Tulua.

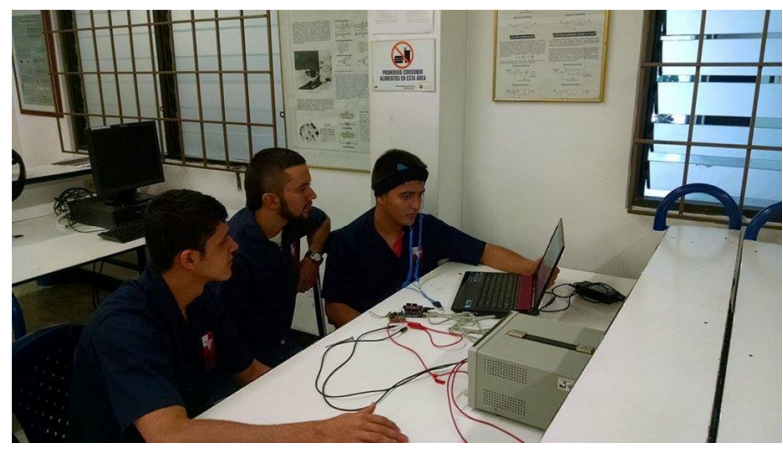


Figura 10. Circuito funcionando.

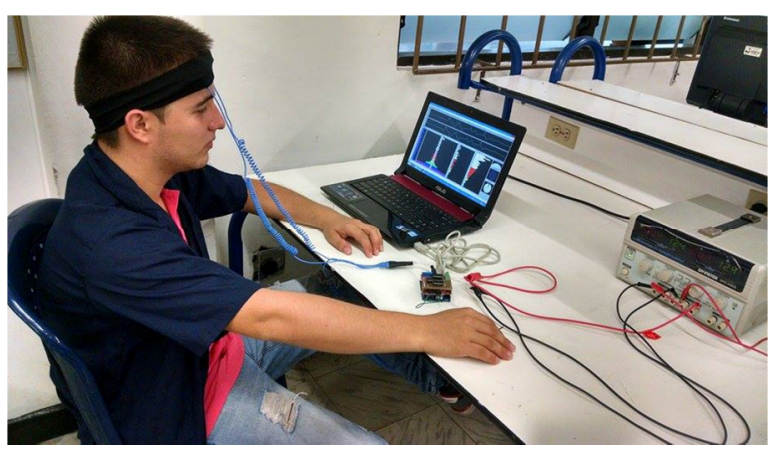

Figura 11. Usuario con el hardware haciendo pruebas.

\section{Aplicaciones posibles}

\section{a. Control}

Las interfaces cerebro computador tienen múltiples campos de acción si se pueden integrar con otras herramientas como la inteligencia artificial.

Los campos de acción en control, pueden incluir el desarrollo de videojuegos, sistemas de encendido de equipos electrónico o generar acciones de control según estados mentales específicos.

\section{b. Medicina}

Los valores correspondientes a las frecuencias de las ondas mentales, pueden servir para medir estados de estrés y concentración que pueden ser utilizados para ser acompañados de protocolos médicos que permitan el diagnóstico de enfermedades.

Es posible medir con estas señales los niveles de atención de los usuarios, además de diferentes actividades de las regiones cerebrales, según la ubicación del electrodo de entrada.

\section{CONCLUSIONES}

- Se logró identificar una alternativa económica de incursión en una tecnología emergente como es la de las interfaces cerebro - computador.

- A pesar de que las señales capturadas, se reciben con algo de ruido, este no afecta considerablemente la medición y es posible utilizarse en entornos académicos.

- $\quad$ Es posible la operación interdisciplinaria de los estudiantes de programas académicos de tecnología e ingeniería, para desarrollar tecnologías de vanguardia.

\section{REFERENCIAS}

[1]. Introducción a la bioingeniería, serie mundo electrónico. Marcombo S.A, 1988

[2]. LLAMOSA RINCÓN, LUÍS ENRIQUE. Diseño de canales para la detección de biopotenciales, Universidad Tecnológica de Pereira, 2005.

[3]. GUYTON ARTHUR C. Tratado de fisiología medica, 11 edición, Elsevier España, 2006.

[4]. KAMEN, EDWARD W, HECK, BONNIE S. Fundamentals of signals and Systems using the web and Matlab, Prentice hall, 2007

[5]. VÉLEZ ÁNGEL, PAULO ANDRÉS, SALDARRIAGA VILLAFAÑE, HAEDERS. Clasificación básica de neuroseñales. Universidad tecnológica de Pereira, facultad de ciencias básicas. Pereira, 2010. 